\title{
Risks of Having Gestational Diabetes in The Future For Mothers and Their Offsprings
}

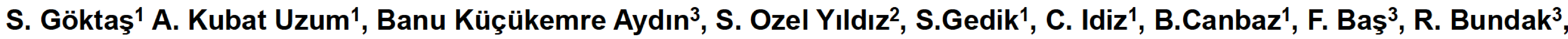 \\ N.Dinccag ${ }^{1}$, \\ 1. Istanbul University, Istanbul Faculty of Medicine, Endocrinology and Metabolism Division, Istanbul-Turkey \\ 2. Istanbul University, Istanbul Faculty of Medicine, Biostatistics Department, Istanbul-Turkey \\ 3, Istanbul University, Istanbul Faculty of Medicine, Paediatric Endocrinology and Metabolism Division, İstanbul-Turkey
}

\section{OBJECTIVES}

Gestational diabetes(GDM) has an important short and longterm health consequences for both the mother and her offspring. The aim of this study was to evaluate current metabolic status of women with GDM history and their offsprings approximately at $10^{\text {th }}$ year after delivery.

\section{METHODS}

24 mothers who had GDM ten years before (mean age $41.08 \pm 4.13 \mathrm{yrs}$; body mass index(BMI) $30.3 \pm 5.9$ ) and their children (11 female,13 male; mean age of $11.0 \pm 1.9 \mathrm{yrs}$ ) were enrolled into the study. To evaluate current metabolic status of mothers, $75 \mathrm{~g}$ OGTT was performed and according to ADA criteria they were classified into two groups as normal glucose tolerance (group 1:NGT) and glucose intolerance (Group 2:IGT and/or DM). Outcomes of fetal determinants were recorded and current clinical status and laboratory features of offsprings were checked and evaluated if there would be any associations with mothers's metabolic status

\begin{tabular}{|c|c|c|c|c|}
\hline \multirow{3}{*}{$\begin{array}{l}\text { Women } \\
\text { (after } 11 \pm 2 \text { years from } \\
\text { delivery }\end{array}$} & & Group I & $\begin{array}{c}\text { Group II } \\
\text { (IFG+IGT/DM) }\end{array}$ & P value \\
\hline & $\mathrm{N}(\%$ & $14(58.5)$ & $3(12.5) / 7(29.2)$ & \\
\hline & $\begin{array}{l}\text { At diagnosis } \\
\text { OGTT } \\
1 \text { st hour } \\
\text { 2nd hour } \\
3 \text { rd hour }\end{array}$ & $\begin{array}{l}100.7 \pm 30.8 \\
186.6 \pm 86.3 \\
160.4 \pm 14.2 \\
128.5 \pm 35.0\end{array}$ & $\begin{array}{l}122.5 \pm 32.8 \\
254.1 \pm 57.1 \\
250.3 \pm 70.6 \\
161.8 \pm 69.2\end{array}$ & $\begin{array}{c}0.08 \\
0.04 \\
0.005 \\
0.5\end{array}$ \\
\hline \multirow[t]{4}{*}{ Fetus of birth } & Length $(\mathrm{cm})$ & $50.7 \pm 2.8$ & $48.9 \pm 2.2$ & 0.1 \\
\hline & Length SDS & $0.76 \pm 1.3$ & $0.83 \pm 1.04$ & 0.4 \\
\hline & Weight (kg) & $3625.6 \pm 680$ & $3197.6 \pm 608$ & 0.08 \\
\hline & Weight SDS & $1.2 \pm 1.4$ & $0.7 \pm 1.7$ & 0.9 \\
\hline \multirow{11}{*}{$\begin{array}{l}\text { Final status of } \\
\text { adolescents }\end{array}$} & Age & & & \\
\hline & Length $(\mathrm{cm})$ & $147.6 \pm 11.5$ & $146.7 \pm 13.0$ & 0.8 \\
\hline & Length SDS & $0.56 \pm 0.78$ & $0.47 \pm 1.35$ & 0.6 \\
\hline & Weight (kg) & $40.94 \pm 10.9$ & $45.9 \pm 19.0$ & 0.7 \\
\hline & Weight SDS & $0.3 \pm 0.8$ & $1.0 \pm 1.9$ & 0.5 \\
\hline & $\mathrm{BMI}\left(\mathrm{kg} / \mathrm{m}^{2}\right)$ & $18.4 \pm 3.1$ & $20.6 \pm 5.7$ & 0.7 \\
\hline & Waist circumference & $67.67 \pm 12.2$ & $71.95 \pm 10.1$ & 0.3 \\
\hline & Glucose (mg/dL) & $92.5 \pm 9.0$ & $92.0 \pm 6.5$ & 0.8 \\
\hline & Insulin ( $\mathrm{mlU} / \mathrm{mL})$ & $7.3 \pm 3.7$ & $9.4 \pm 7.9$ & 0.6 \\
\hline & FBG / FBI & & & 0.03 \\
\hline & HbA1C (\%) & $5.49 \pm 0.5$ & $5.40 \pm 0.1$ & 0.8 \\
\hline
\end{tabular}

\section{CONCLUSIONS}

Frequency of glucose intolerance may be increased in the follow-up years; offsprings of mothers having prediabetes may have great risk for obesity and insulin resistance in the future; especially at pubertal period. Those adolescents should be identified and efforts should be done to prevent development of metabolic syndrome and lifestyle should be planned to preserve beta cell function of the pancreas before puberty.

\section{RESULTS}

$41.6 \%$ of mothers had any degree of glucose intolerance at tenth year after delivery. As we concluded fetal determinants in groups, measurements of birth weight(gr), lenght $(\mathrm{cm})$ were found as $3625.6 \pm 680$ vs $3197.6 \pm 608$; $147.6 \pm 11.5$ vs $146.7 \pm 13.0$, respectively. As we considered daily status of children, their BMI and waist circumferences were observed as $18.4 \pm 3.1$ vs $20.6 \pm 5.7 \mathrm{~kg} / \mathrm{m}^{2}$ and $67.7 \pm 12.2$ vs $71.9 \pm 10.1$ $\mathrm{cm}$, respectively $(p>0.05)$. Although the difference was not significant, waist circumference and central obesity of offsprings in group 2 was found higher. Also results of fasting glucose and insulin levels of children in group 2 were higher than the others; but difference was not statistically significant. Five of them had lipid profile changes.

\section{References}

1. Hatipoglu N, Mazicioglu MM, Poyrazoglu S, et al. Waist circumference percentiles among Turkish children under

the age of 6 years. Eur J Pediatr (2013) 172:59-69. DOI 10.1007/s00431-012-1822-5

2.Boney CM, Verma A, Tucker T, et al. Metabolic Syndrome in Childhood: Association With Birth Weight, Maternal Obesity, and Gestational Diabetes Mellitus. PEDIATRICS Vol. 115 